\title{
Fano Resonance of the Symmetry-Reduced Metal Bar Grating Structure
}

\author{
Suxia Xie, Zhijian Li, Renlong Zhou, Jie Zhan, Qiong Liu, Lingxi Wu, and Bingju Zhou \\ School of Physics and Electronic Science, Hunan University of Science and Technology, Xiangtan 411201, China \\ Correspondence should be addressed to Suxia Xie; xiesuxia2013@163.com
}

Received 4 March 2014; Accepted 9 August 2014; Published 16 October 2014

Academic Editor: Christian Brosseau

Copyright (C) 2014 Suxia Xie et al. This is an open access article distributed under the Creative Commons Attribution License, which permits unrestricted use, distribution, and reproduction in any medium, provided the original work is properly cited.

\begin{abstract}
We demonstrate that Fano resonance and even multipole Fano resonance can be obtained in a symmetry-reduced structure composed of gold bars with different bar sizes or bar shapes on a layer of dielectric. There is a transparency window opened within the frequency region of the absorptive dipole resonance by metallic bars, as long as the narrow grating waveguide mode induced by reducing symmetry is coincided in spectrum with the dipole resonance such that a destructive interference happens between these two resonant modes. Line shape of the transmission spectra of the nanostructure can be modulated effectively by changing the size or shape of the series of metal bars. The results found can be useful in the design of novel optical device.
\end{abstract}

\section{Introduction}

Fano resonance as a coherent phenomenon has emerged as a common characteristic of complex, coupled plasmon system [1]. This effect is important in the line shape engineering, and the frequency tunability of plasmonic nanosystems has been well established. Fano resonance can be obtained in materials with negative permeability $(\mu<0)$ and positive dielectric permittivity $(\varepsilon>0)$, even a material with negative refractive index, where both $\varepsilon$ and $\mu$ are negative [2]. Fano resonance can also be obtained in anisotropic materials, where the intensity of the surface plasmon resonance can be greatly enhanced. Much of the original work on plasmonic Fano resonance was carried out on metallic arrays. The broad resonance providing the continuum for the narrow Fano resonance is a strongly radiative collective dipolar mode formed from a coupling of the plasmons on the individual array elements.

Metallic structures which lead to Fano resonance are divided into three kinds according to structure. The first are the plasmonic nanostructures such as dolmen-type slab arrangements [3], the nonconcentric ring/disk cavity [46], symmetry breaking ring/disk [7], and finite clusters of plasmonic nanoparticles $[5,8]$. The second are the metallic photonic crystals; for example, an array of gold nanowires placed on a single-mode slab waveguide exhibits a Fano resonance in extinction in transverse electric polarization owing to coupling between the array and the waveguide. The last are the metamaterials. Fano resonance in metamaterials was observed for the first time in asymmetrically split-ring arrays [9]. Then polarization sensitive Fano resonance linked to strong optical activity and circular dichroism in the microwave and optical parts of the spectrum can be engaged through constructing a chiral arrangement of the metamaterial array with respect to the incident electromagnetic wave [10]. A Fano metamaterial with polarization-insensitive resonance, with behavior independent of incidence direction of light, has also been introduced [11]. Fano resonances have recently been observed in a superconducting metamaterial, promising extremely high-Q modes [12]. Fano resonance is associated with the coherent interference of "bright" and "dark" hybridized plasmon modes [1, 13-15]. Bright plasmon modes possess finite dipole moments, where their resonance is spectrally broadened due to radiative damping. In contrast, dark plasmon modes possess zero or nearly zero dipole moments, do not couple efficiently to light, and are therefore not broadened. The coupling between bright and dark plasmon modes occurs through the electromagnetic near-field and can be controlled using symmetry breaking [16-19]. As Fano resonances arise from the interference 
between two or more oscillators, they possess an inherent sensitivity to changes in geometry or local environment: small perturbations can induce dramatic resonance or line shape shifts. This property renders Fano resonant media particularly attractive for a range of applications, such as the development of chemical or biological sensors.

In this paper, we investigate a Fano resonance planar structure composed of a gold-bar grating placed on a dielectric layer. A distinct structure for the periodic gold grating proposed in this work is the symmetry-reduced arrangement. It is demonstrated that Fano resonance even multipole Fano resonance appears in the transmission spectrum, and there is a transparency window opened within the frequency region of the absorptive dipole resonance by metallic bars, as long as the narrow grating waveguide mode induced by reducing symmetry is coincided in spectrum with the dipole resonance such that a destructive interference happens between these two resonant modes. Line shape of the transmission spectra of the nanostructure can be modulated effectively by changing the size or shape of the series of metal bars. For example, little variation of any bar's size and location in one lattice may lead to a transparency window in the transmission spectrum, and the width and wavelength of the transparency window can be modulated by each bar's size and location in the lattice obviously. Additionally, more modulating factor is introduced by the hollow bar in the lattice; the introduction of the inner size of the metallic bar may lead the line shape of the transmission spectra to be modulated better. The results may be helpful for the design of new optical device.

\section{Material and Methods}

The analyzed structure is presented in Figure 1. In our 2D FDTD calculations $[20,21]$, perfectly matched layer boundary conditions [22] are used at the top and bottom, and periodic boundary conditions are used on the left and right sides of the lattice due to the periodicity of the system. We simulate the structure with a computational window of $L_{x} \times$ $L_{y}=700 \mathrm{~nm} \times 2000 \mathrm{~nm}$, where the structure in the $z$ direction is uniform and infinite. The structure is periodic and the periodicity is $p=700 \mathrm{~nm}$, and there are three gold bars contained in the lattice. We send a Gaussian single pulse of light with a wide frequency profile and an incidence angle of $90^{\circ}$ illuminating the metal bar grating from the bottom of the lattice in the $x y$ cross section. Parameters of the Au grating are denoted as the widths of the three bars in the lattice: $s_{1}, s_{2}, s_{3}$, in which $s_{1}$ and $s_{3}$ are fixed as $100 \mathrm{~nm}$. The shape of the middle bar can be set as a tube, and the inner size of the tube is denoted as $s_{22}$. Gaps between the three bars are denoted as $l_{1}, l_{2}$. The offset of localization of the middle bar from the center of the lattice in $x$ direction is denoted as $c$. The thickness of the dielectric under the Au bar array is fixed as $h=200 \mathrm{~nm}$, and relative permittivity is set as $\varepsilon_{r}=3$.

\section{Results and Discussions}

For the symmetric grating array with identical bar size and bar gap, only the dipole plasmon resonance can be excited

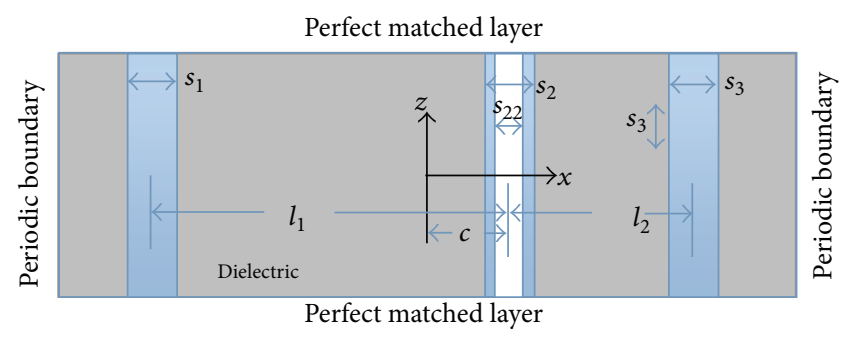

(a)

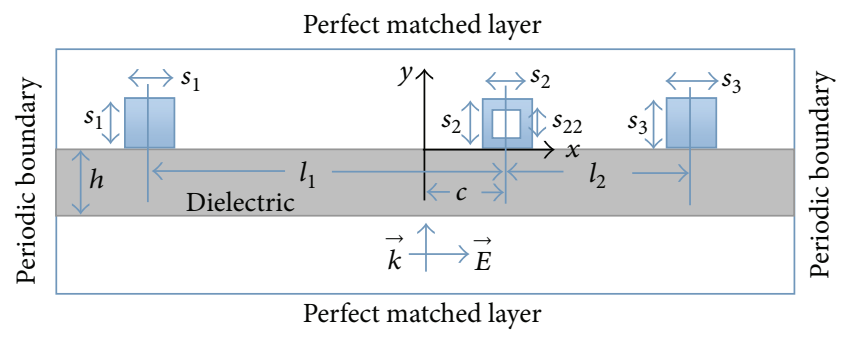

(b)

FIGURE 1: $x-y$ cross section of one lattice containing three gold bars of the gold periodic bar array on a layer of dielectric for the FDTD simulations. Parameters are defined in the texts.

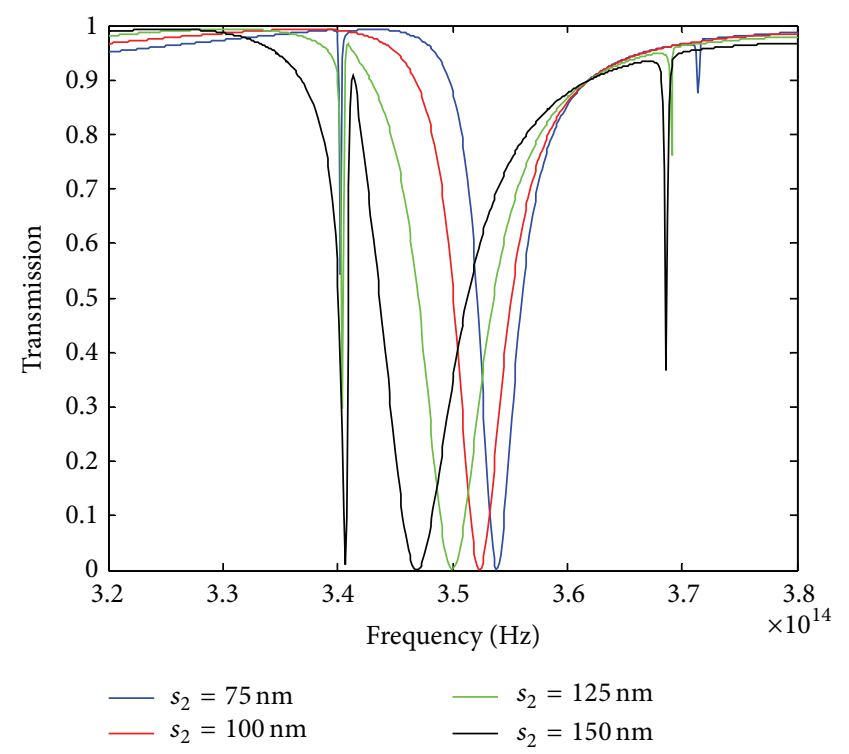

FIGURE 2: The transmission spectra of a lattice contain 3 bars of a periodic bar array with size of the center bar of the lattice $s_{2}=75 \mathrm{~nm}$, $100 \mathrm{~nm}, 125 \mathrm{~nm}$, and $150 \mathrm{~nm}$, respectively, size of the other two bars of the array is $s_{1}=s_{3}=100 \mathrm{~nm}$, distance between bars is $700 \mathrm{~nm}$, and other parameters are $s_{22}=0, c=0$, respectively.

by incident waves, corresponding to only one transmission dip (as shown in Figure $2\left(s_{1}=100 \mathrm{~nm}\right)$ ), additionally, there is only one transmission dip, whatever the bar size and gap size is. In contrast, it is interesting to find that there will be a transmission dip at a lower frequency around the dipole resonant transmission dip for the symmetry-reduced bar grating structure with size of the center bar of the lattice getting smaller or larger than the other two. The larger 
the size of the center bar deviates from the size of other bars, the more obvious the new transmission dip is. Along with offset of the size of the center bar increasing, the new transmission dip gets deeper, which means that its intensity gets stronger; meanwhile, its full width at half maximum gets larger. The new transmission dip with an asymmetrical shape can be attributed to the Fano resonance associated with the coupling of transversal surface plasmon resonance mode and localized surface plasmon resonance mode. In addition, with the increasing of size of center bar of the lattice, center frequency of the new transmission dip hardly moves, but the original transmission dip red shifts obviously accompanied by the increase of full width at half maximum. Besides, the transmission peak between two transmission dips becomes narrow, which forms into a transparency window, as an EITlike phenomenon when the destructive interference happens between a broad resonance (dipole mode) and a narrow one (usually a subradiant mode). Here the subradiant mode resonance comes from the grating waveguide structure. When light is illuminated on the periodic metal nanobar grating, there is surface plasmon dipole mode excited, but there is no grating waveguide mode excited for the symmetric grating configuration with unified bar size and bar gap; however, the numerical result indicates that a symmetry-reduced periodic metal bar grating can make it an excitable mode, and this interesting asymmetry-induced resonance is in accordance with recent literatures [23-25]. Additionally, the dark mode excitation by the way of breaking or reducing symmetry can be feasible instead of the one by the plasmon-coupling excitation of a radiant resonant mode [26]. Besides, another shallow transmission dip appears at the higher frequency range, and its intensity gets stronger as the size of the center bar increases. This transmission dip may also be attributed to the Fano resonance. As a result, multiple Fano resonances can be obtained and modulated effectively by changing the size of one of the three bars in the lattice.

We also simulate the transmission characteristics of the metal grating structure as size varies of the side bars in the lattice (as shown in Figure 3). It is shown that the results are the same as those in Figure 2 absolutely.

Figure 4 shows the dependence of the transmission spectra of lattice containing 3 bars of a periodic bar array with localization of the center bar; when the center bar is set at the center between the left and the right bar, there is a wide transmission dip in the spectrum associated with the surface plasmon dipole mode. When the center bar moves along the $x$ direction, a new transmission dip appears at a lower frequency, which can be attributed to the Fano resonance. With the center bar of the lattice moving on, the Fano resonance mode and the original transmission dip gets wider, but central frequencies of both resonance modes do not shift obviously. Additionally, if the center bar moves further, higher multipolar surface modes can also interfere with the broad dipole mode and generate higher-order Fano resonances when the size of the middle metal bar is increased. In Figure 4, the appearance of the octupolar Fano resonance resulting from these higher order interactions is shown.

In Figure 5, the calculated transmission spectra of the lattice containing three metal bars with center bar a hollow

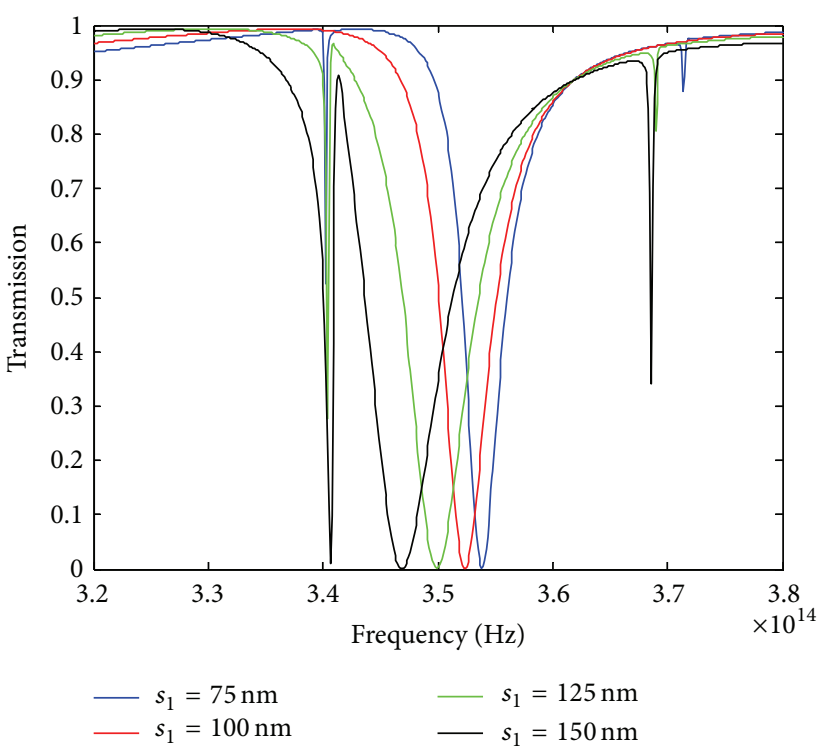

FIgURE 3: The transmission spectra of a lattice contain 3 bars of a periodic bar array with size of the left bar of the lattice $s_{1}=75 \mathrm{~nm}$, $100 \mathrm{~nm}, 125 \mathrm{~nm}$, and $150 \mathrm{~nm}$, respectively, size of the other two bars of the array is $s_{2}=s_{3}=100 \mathrm{~nm}$, distance between bars is $700 \mathrm{~nm}$, and other parameters are $s_{22}=0, c=0$, respectively.

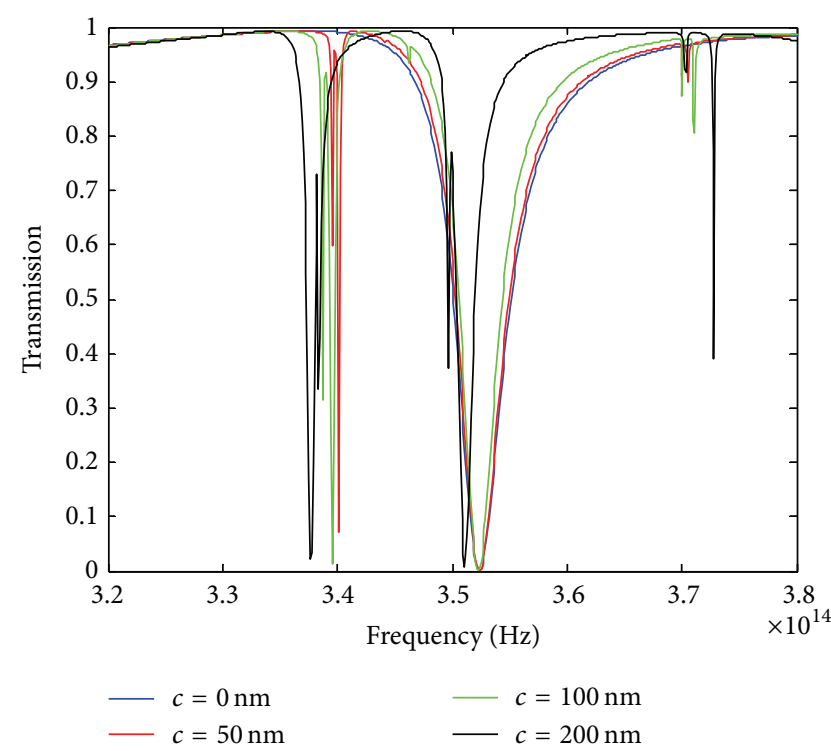

FIGURE 4: The transmission spectra of a lattice contain 3 bars of a periodic bar array with offset from center of the lattice to the localization of the center bar in $x$ direction is $c=0,50 \mathrm{~nm}, 100 \mathrm{~nm}$, and $200 \mathrm{~nm}$, respectively, size of bars of the array is $s_{1}=s_{2}=s_{3}=$ $100 \mathrm{~nm}, s_{22}=0$, and distance between bars is $700 \mathrm{~nm}$, respectively.

tube are shown. It is shown that there is only one transmission dip (the dipole mode) of the inner size of the center bar less than $50 \mathrm{~nm}$. The dipole mode transmission dip red shifts slowly when $s_{22}$ increases; for example, central frequency moves $0.0012 \times 10^{14} \mathrm{~Hz}$ when inner size of tube rises from $50 \mathrm{~nm}$ to $80 \mathrm{~nm}$. As the inner size of the tube increases when 


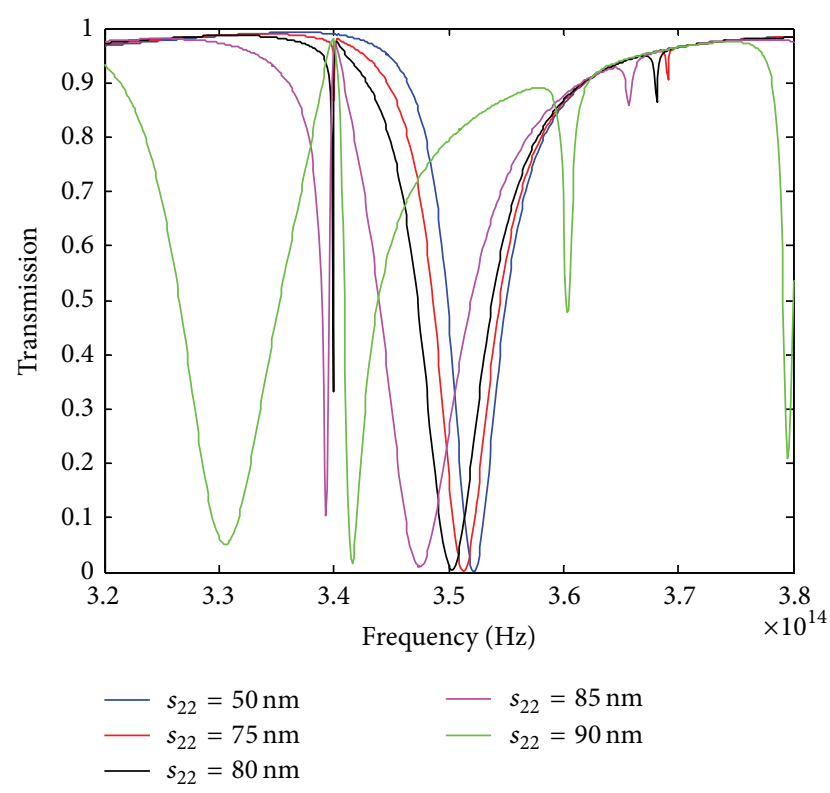

FIGURE 5: The transmission spectra of a lattice contain 3 bars of a periodic bar array, and the middle bar is a hollow tube with inner size $s_{22}=50 \mathrm{~nm}, 75 \mathrm{~nm}, 80 \mathrm{~nm}, 85 \mathrm{~nm}$, and $90 \mathrm{~nm}$, respectively, size of bars of the array is $s_{1}=s_{2}=s_{3}=100 \mathrm{~nm}$, distance between bars is $700 \mathrm{~nm}$, and offset is $c=0$, respectively.

$s_{22}$ is small, the transmission dip moves much faster; it shifts rapidly for $0.1044 \times 10^{14} \mathrm{~Hz}$ as inner size of tube increases from $80 \mathrm{~nm}$ to $90 \mathrm{~nm}$ when the inner size is large. As the inner size of the center bar increases, a new shallow transmission dip with an intensity appears $\left(0.8663\right.$ at $\left.s_{22}=75 \mathrm{~nm}\right)$; with the inner size increasing continually, the new transmission dip intensity is enhanced dramatically $\left(0.0514\right.$ at $s_{22}=$ $90 \mathrm{~nm}$ ). Moreover, along with the inner tube size increasing, both the full width at half maximum and central frequency change quickly. Central frequencies of both transmission dips red shift obviously along with $s_{22}$ increasing; especially, the larger the $s_{22}$ size is, the faster the central frequency moves. For example, the central frequency moves from $3.3932 \times$ $10^{14} \mathrm{~Hz}$ to $3.3056 \times 10^{14} \mathrm{~Hz}$ by a $0.0876 \times 10^{14} \mathrm{~Hz}$, with the inner tube size rising from $85 \mathrm{~nm}$ to $90 \mathrm{~nm}$. Additionally, the transparency window between the two transmission dips is associated with the EIT-like phenomenon. The central frequency of the transmission peak hardly moves around 3.4 $\times 10^{14} \mathrm{~Hz}$ with $s_{22}$ increasing with a value close to 1 . Additionally, along with the increase of the inner tube size, there is new shallow transmission dip emerging at the higher frequency region, and it may also be attributed to the Fano resonance. So it is improved that Fano resosonance even multiple Fano resonance can be obtained in a periodic metal structure with a series hollow bars, which can be modulated effectively by the dimension of the inner bar size. It is shown in Figure 5 that the dipole mode transmission dip is widened along with the inner size of the tube in the lattice, and it is found that it gets narrow when $s_{22}$ increases from $85 \mathrm{~nm}$ to $90 \mathrm{~nm}$. We can understand it like this: the dipole mode transmission dip red shifts with the inner size of the tube increasing, but

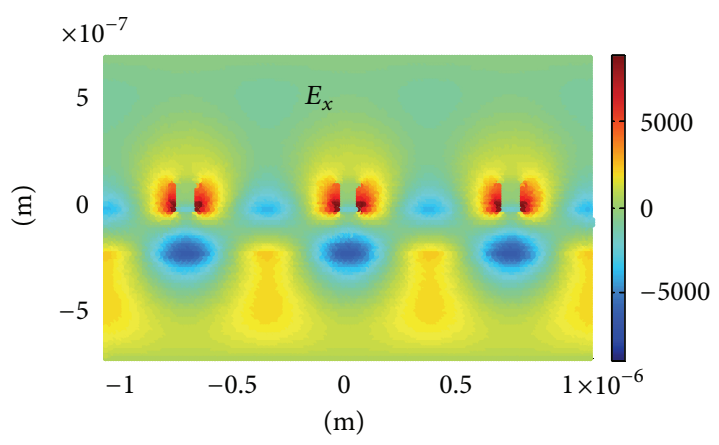

(a)

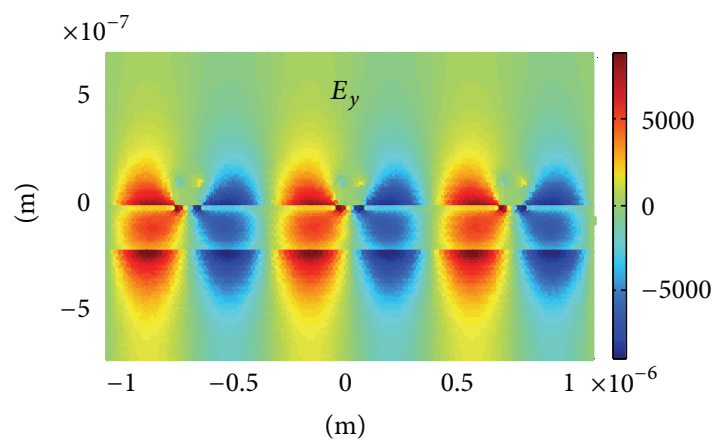

(b)

Figure 6: Cross sections of the spatial distribution $\vec{E}_{x}$ and $\vec{E}_{y}$ of the field intensity of the dipole mode resonance at the center peak frequency $f=3.5240 \times 10^{14} \mathrm{~Hz}$ in the transmission spectrum of the symmetric periodic metal bar array with bar size $s_{1}=s_{2}=s_{3}=$ $100 \mathrm{~nm}$, bar gap $l_{1}=l_{2}=700 \mathrm{~nm}$, inner size of the middle bar $s_{22}=$ 0 , and offset $c=0$.

the central frequency of the transmission peak between the new transmission dip and the original one stays at the same location all the time, which leads to the result that the left part of the dipole mode transmission dip red-shift is restricted, so the line width is narrowed. It is clear that the bright dipole mode can be transferred into the subradiant waveguiding mode as long as the grating symmetry is reduced, resulting in a transparency narrow band, as shown in the spectra between the dipole mode transmission dip and the Fano resonance (the new transmission dip at the left of the dipole mode) in Figures 2 and 5. It can be understood physically in this way that the destructive interference happens predominantly so that the electromagnetic energy of the dipole resonance is totally transferred to the grating waveguide resonance, which apparently contributes to the transparency window.

To explicitly verify the dipole resonance (transmission dip) and the symmetry-reduction induced Fano resonance (the new transmission dip), the resonant E-filed distributions are plotted. It is interesting to found that strong E-field distribution is localized between the bars for the symmetric gold bar array with unified bar size, bar gap, and bar shape, due to the standing wave induced of the dipole mode (as shown in Figure 6).

It can be obtained that dipole resonance is excited in the symmetry configuration with equal bar size and equal 


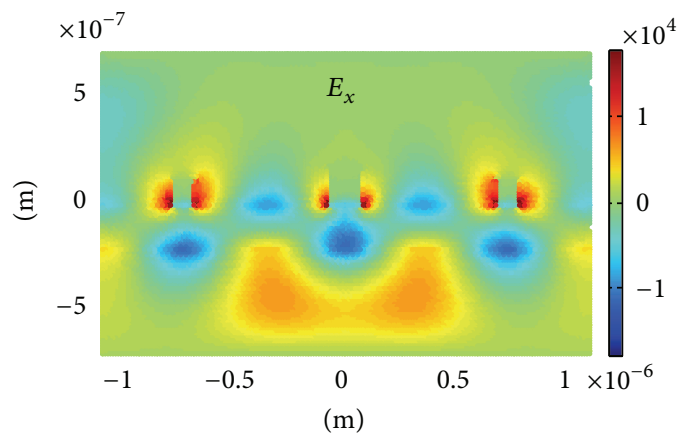

(a)

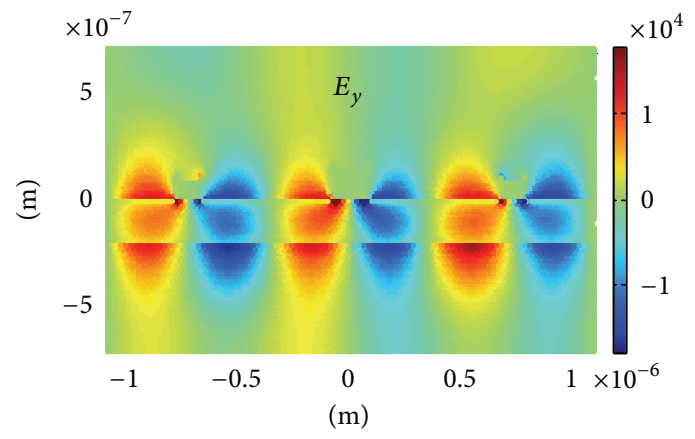

(b)

FIGURE 7: Cross sections of the spatial distribution $\vec{E}_{x}$ and $\vec{E}_{y}$ of the field intensity of the dipole mode resonance at the center peak frequency $f=3.4688 \times 10^{14} \mathrm{~Hz}$ in the transmission spectrum of the symmetric-reducing periodic metal bar array with bar size $s_{1}=s_{3}=100 \mathrm{~nm}$, $s_{2}=150 \mathrm{~nm}$, bar gap $l_{1}=l_{2}=700 \mathrm{~nm}$, inner size of the middle bar $s_{22}=0$, and offset $c=0$.

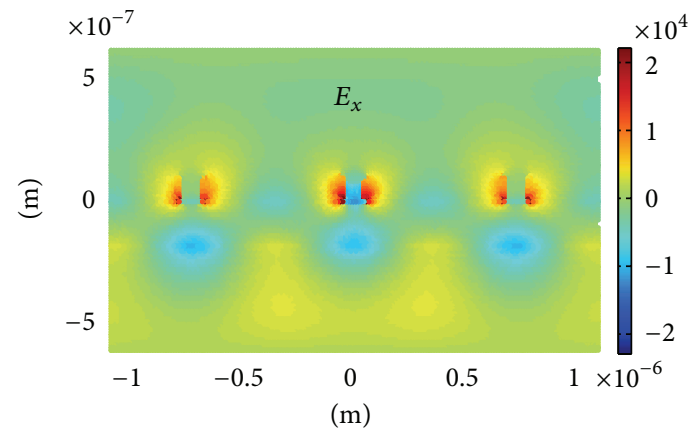

(a)

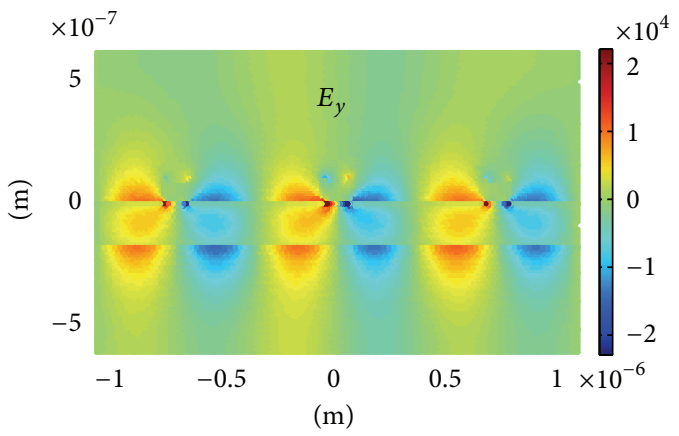

(b)

FIGURE 8: Cross sections of the spatial distribution $\vec{E}_{x}$ and $\vec{E}_{y}$ of the field intensity of the dipole mode resonance at the center peak frequency $f=3.4784 \times 10^{14} \mathrm{~Hz}$ in the transmission spectrum of the symmetric-reducing periodic metal bar array with bar size $s_{1}=s_{2}=s_{3}=100 \mathrm{~nm}$, inner bar size of the middle bar $s_{22}=85 \mathrm{~nm}$, bar gap $l_{1}=l_{2}=700 \mathrm{~nm}$, and offset $c=0$.

bar gap (as shown in Figure 6). In the symmetry-reduced configuration with the size or inner size of the middle bar increasing in the lattice, dipole modes distributions are changed, the intensity of the electric field localized around the metal bars is weakened dramatically (as shown in Figures 7 and 8), due to the standing wave induced in the structure, which is corresponding the red-shift of the transmission dip in Figures 2 and 5 in the symmetry reduced configuration.

When the plane wave is incident normally to the symmetric metal bar grating, the electric field distributions in the bar grating should have the same vector direction (in phase) and hence it does not excite the transversal grating waveguide mode. However, for the symmetry-reduced case the standing wave of the grating waveguide mode is excitable since the asymmetric bar sizes can break the synchronized phase of the plane wave impinging onto the metal surface; as a result, nodes of the transversal standing wave are formed. The waveguide mode interferes destructively with the channel of absorptive dipole resonance and consequently a transparency window is obtained, which comes from the low-loss nature for the grating modulated waveguiding mode within the dielectric layer. In other words, the Fano resonance mode emerging at the left of the dipole mode transmission dip may be attributed to the coupling of the dipole mode and the waveguide mode, which can be seen clearly in the field distributions in Figures 9 and 10.

\section{Conclusion}

In conclusion, it is found that under the condition of reducing the grating symmetry by altering the size or shape of a series of metal bars in the nanostructure, Fano resonance or even multipole Fano resonance can be obtained in the transmission spectra. A transparency window associated with the EIT-like phenomenon is obtained between the dipole mode and the Fano resonance. Line shape of the transmission spectra of the nanostructure can be modulated effectively by changing the size or shape of the series of metal bars. The results may be helpful for the design of new optical device.

\section{Conflict of Interests}

The authors declare that there is no conflict of interests regarding the publication of this paper. 


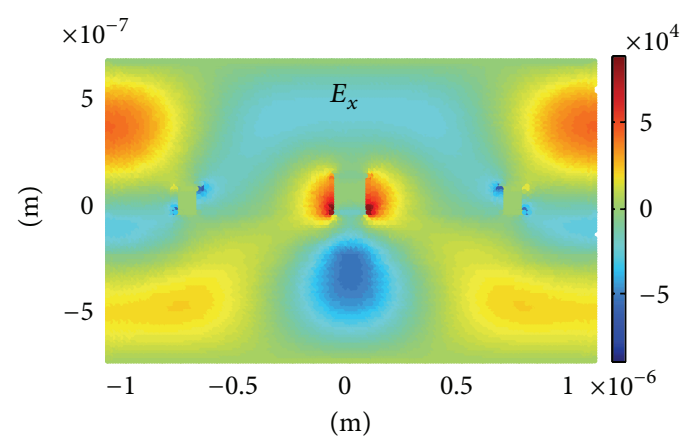

(a)

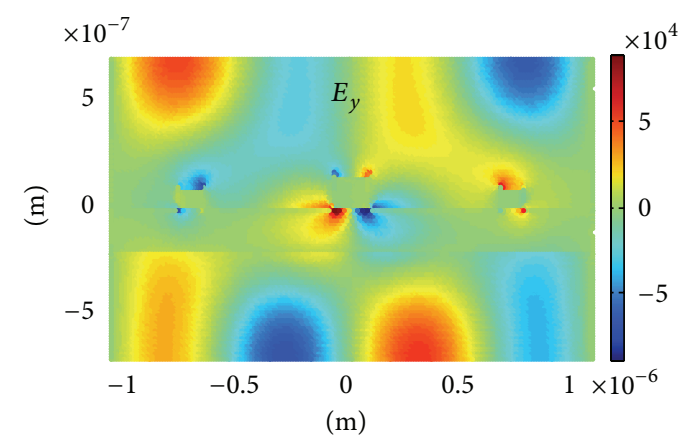

(b)

FIgURE 9: Cross sections of the spatial distribution $\vec{E}_{x}$ and $\vec{E}_{y}$ of the field intensity of the Fano resonance at the center peak frequency $f=3.4064 \times 10^{14} \mathrm{~Hz}$ in the transmission spectrum of the symmetric-reducing periodic metal bar array with bar size $s_{1}=s_{3}=100 \mathrm{~nm}$, $s_{2}=150 \mathrm{~nm}$, and bar gap $l_{1}=l_{2}=700 \mathrm{~nm}$, inner bar size $s_{22}=0$, and offset $c=0$.

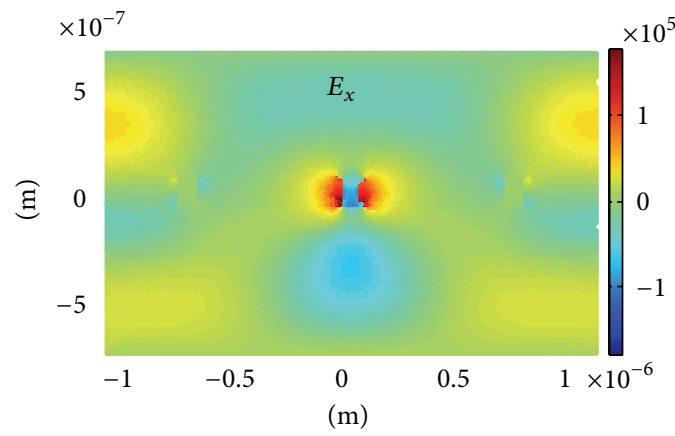

(a)

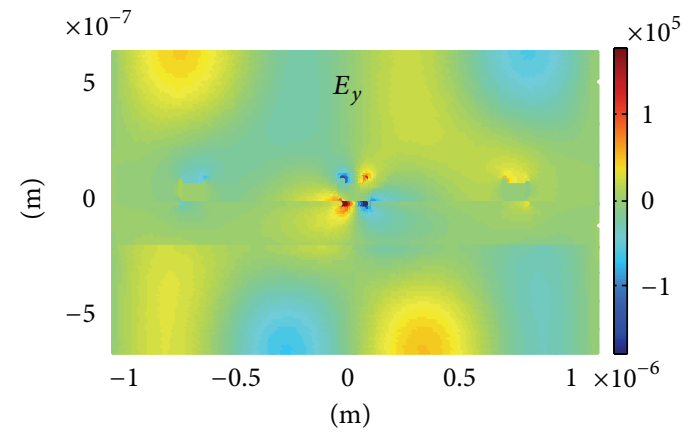

(b)

FIGURE 10: Cross sections of the spatial distribution $\vec{E}_{x}$ and $\vec{E}_{y}$ of the field intensity of the dipole mode resonance at the center peak frequency $f=3.3932 \times 10^{14} \mathrm{~Hz}$ in the transmission spectrum of the symmetric-reducing periodic metal bar array with bar size $s_{1}=s_{2}=s_{3}=100 \mathrm{~nm}$, inner size of the middle bar $s_{22}=85 \mathrm{~nm}$, and bar gap $l_{1}=l_{2}=700 \mathrm{~nm}$, and offset $c=0$.

\section{Acknowledgments}

This work was supported by National Natural Science Foundation of China (under Grant 11304094), Hunan Provincial Natural Science Foundation of China (under Grant 12JJB001), the Scientific Research Fund of Hunan Provincial Education Department (under Grant 13C322), the National Natural Science Foundation of China (under Grant 11247003), Hunan Provincial Department of Education Science Research Key Project of China (under Grant 12A045), Hunan University of Science and Technology Key Bidding Tesearch Topic (under Grant G31102), and Open Project of National Laboratory for Infrared Physics, Chinese Academy of Sciences (under Grant GJKF.20130027).

\section{References}

[1] B. Luk'Yanchuk, N. I. Zheludev, S. A. Maier et al., "The Fano resonance in plasmonic nanostructures and metamaterials," Nature Materials, vol. 9, no. 9, pp. 707-715, 2010.

[2] N. Liu, T. Weiss, M. Mesch et al., "Planar metamaterial analogue of electromagnetically induced transparency for plasmonic sensing," Nano Letters, vol. 10, no. 4, pp. 1103-1107, 2010.
[3] S. Zhang, D. A. Genov, Y. Wang, M. Liu, and X. Zhang, "Plasmon-induced transparency in metamaterials," Physical Review Letters, vol. 101, Article ID 047401, 2008.

[4] F. Hao, P. Nordlander, Y. Sonnefraud, P. van Dorpe, and S. A. Maier, "Tunability of subradiant dipolar and fano-type plasmon resonances in metallic ring/disk cavities: implications for nanoscale optical sensing," ACS Nano, vol. 3, no. 3, pp. 643-652, 2009.

[5] N. A. Mirin, K. Bao, and P. Nordlander, "Fano Resonances in plasmonic nanoparticle aggregates," Journal of Physical Chemistry A, vol. 113, no. 16, pp. 4028-4034, 2009.

[6] Y. Sonnefraud, N. Verellen, H. Sobhani et al., "Experimental realization of subradiant, superradiant, and fano resonances in ring/disk plasmonic nanocavities," ACS Nano, vol. 4, no. 3, pp. 1664-1670, 2010.

[7] N. Verellen, Y. Sonnefraud, H. Sobhani et al., "Fano resonances in individual coherent plasmonic nanocavities," Nano Letters, vol. 9, no. 4, pp. 1663-1667, 2009.

[8] F. Le, D. W. Brandl, Y. A. Urzhumov et al., "Metallic nanoparticle arrays: a common substrate for both surface-enhanced Raman scattering and surface-enhanced infrared absorption," ACS Nano, vol. 2, no. 4, pp. 707-718, 2008. 
[9] V. A. Fedotov, M. Rose, S. L. Prosvirnin, N. Papasimakis, and N. I. Zheludev, "Sharp trapped-mode resonances in planar metamaterials with a broken structural symmetry," Physical Review Letters, vol. 99, Article ID 147401, 2007.

[10] E. Plum, X.-X. Liu, V. A. Fedotov, Y. Chen, D. P. Tsai, and N. I. Zheludev, "Metamaterials: optical activity without chirality," Physical Review Letters, vol. 102, no. 11, Article ID 113902, 2009.

[11] N. Papasimakis, Y. H. Fu, V. A. Fedotov, S. L. Prosvirnin, D. P. Tsai, and N. I. Zheludev, "Metamaterial with polarization and direction insensitive resonant transmission response mimicking electromagnetically induced transparency," Applied Physics Letters, vol. 94, no. 21, Article ID 211902, 2009.

[12] V. A. Fedotov, A. Tsiatmas, J. H. Shi et al., "Temperature control of Fano resonances and transmission in superconducting metamaterials," Optics Express, vol. 18, no. 9, pp. 9015-9019, 2010.

[13] K. C. Woo, L. Shao, H. J. Chen, Y. Liang, J. F. Wang, and H.Q. Lin, "Universal scaling and fano resonance in the plasmon coupling between gold nanorods," ACS Nano, vol. 5, no. 7, pp. 5976-5986, 2011.

[14] A. Artar, A. A. Yanik, and H. Altug, "Multispectral plasmon induced transparency in coupled meta-atoms," Nano Letters, vol. 11, no. 4, pp. 1685-1689, 2011.

[15] B. Gallinet and O. J. F. Martin, "Ab initio theory of Fano resonances in plasmonic nanostructures and metamaterials," Physical Review B, vol. 83, no. 23, Article ID 235427, 2011.

[16] L. Chuntonov and G. Haran, "Trimeric plasmonic molecules: the role of symmetry," Nano Letters, vol. 11, no. 6, pp. 2440-2445, 2011.

[17] D. E. Gómez, K. C. Vernon, and T. J. Davis, "Symmetry effects on the optical coupling between plasmonic nanoparticles with applications to hierarchical structures," Physical Review B, vol. 81, no. 7, Article ID 075414, 2010.

[18] S. P. Zhang, K. Bao, N. J. Halas, H. X. Xu, and P. Nordlander, "Substrate-induced Fano resonances of a plasmonic nanocube: a route to increased-sensitivity localized surface plasmon resonance sensors revealed," Nano Letters, vol. 11, no. 4, pp. 16571663, 2011.

[19] Z.-J. Yang, Z.-S. Zhang, L.-H. Zhang, Q.-Q. Li, Z.-H. Hao, and Q.-Q. Wang, "Fano resonances in dipole-quadrupole plasmon coupling nanorod dimers," Optics Letters, vol. 36, no. 9, pp. 1542-1544, 2011.

[20] A. Taflove and S. C. Hagness, Computational Electrodynamics: The Finite-Difference Time-Domain Method, Artech, London, UK, 2005.

[21] K. S. Yee, "Numerical solution of initial boundary value problems involving Maxwell's equations in isotropic media," IEEE Transactions on Antennas and Propagation, vol. 14, pp. 302-307, 1966.

[22] J. Berenger, "A perfectly matched layer for the absorption of electromagnetic waves," Journal of Computational Physics, vol. 114, no. 2, pp. 185-200, 1994.

[23] A. Christ, O. J. F. Martin, Y. Ekinci, N. A. Gippius, and S. G. Tikhodeev, "Symmetry breaking in a plasmonic metamaterial at optical wavelength," Nano Letters, vol. 8, no. 8, pp. 2171-2175, 2008.

[24] F. Hao, Y. Sonnefraud, P. van Dorpe, S. A. Maier, N. J. Halas, and P. Nordlander, "Symmetry breaking in plasmonic nanocavities: Subradiant LSPR sensing and a tunable Fano resonance," Nano Letters, vol. 8, no. 11, pp. 3983-3988, 2008.
[25] L. V. Brown, H. Sobhani, J. B. Lassiter, P. Nordlander, and N. J. Halas, "Heterodimers: plasmonic properties of mismatched nanoparticle pairs," ACS Nano, vol. 4, no. 2, pp. 819-832, 2010.

[26] Z.-G. Dong, H. Liu, M.-X. Xu et al., "Role of asymmetric environment on the dark mode excitation in metamaterial analogue of electromagnetically-induced transparency," Optics Express, vol. 18, no. 21, pp. 22412-22417, 2010. 

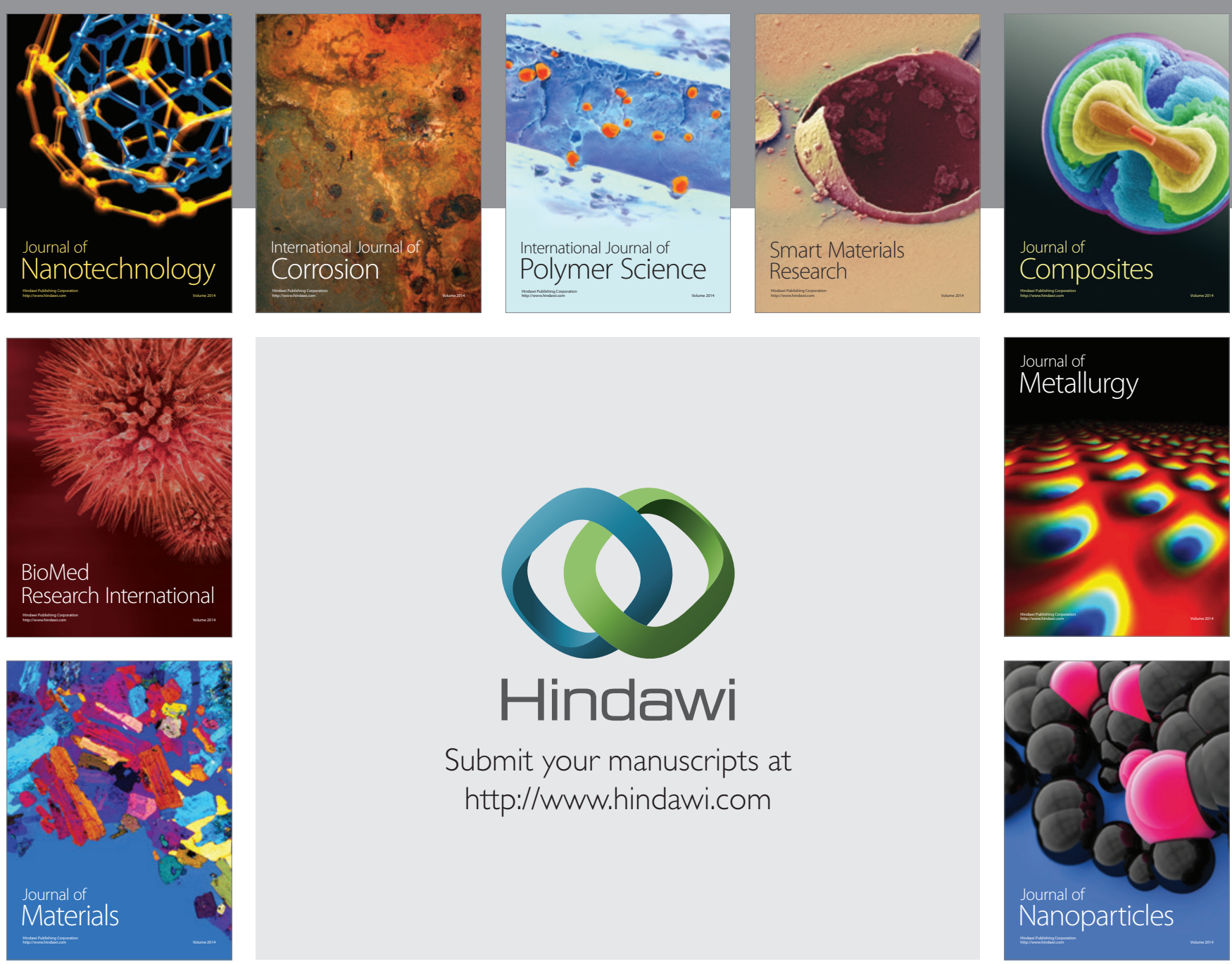

Submit your manuscripts at http://www.hindawi.com
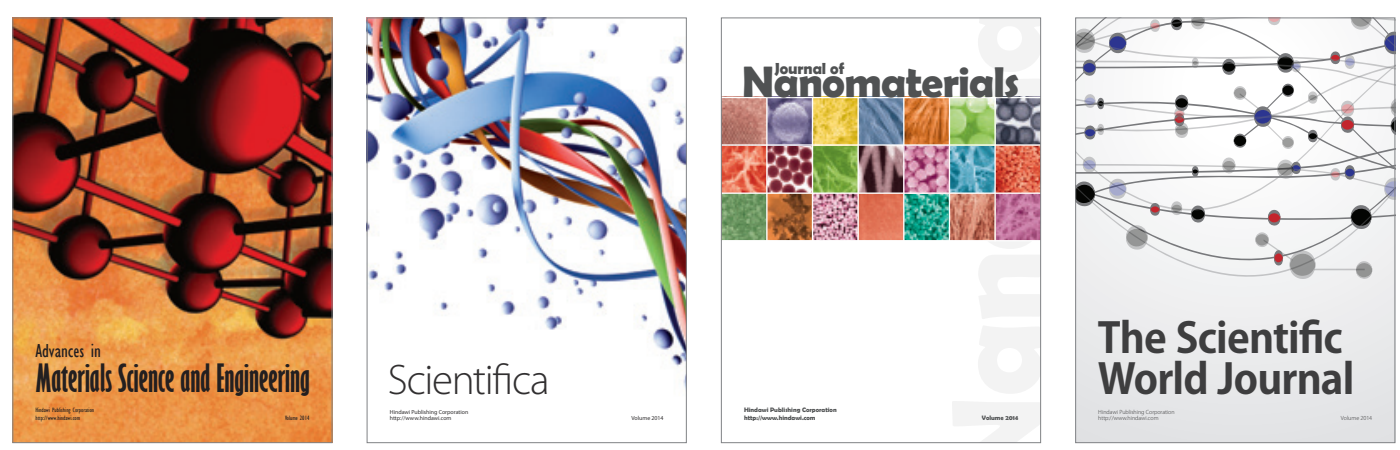

\section{The Scientific World Journal}
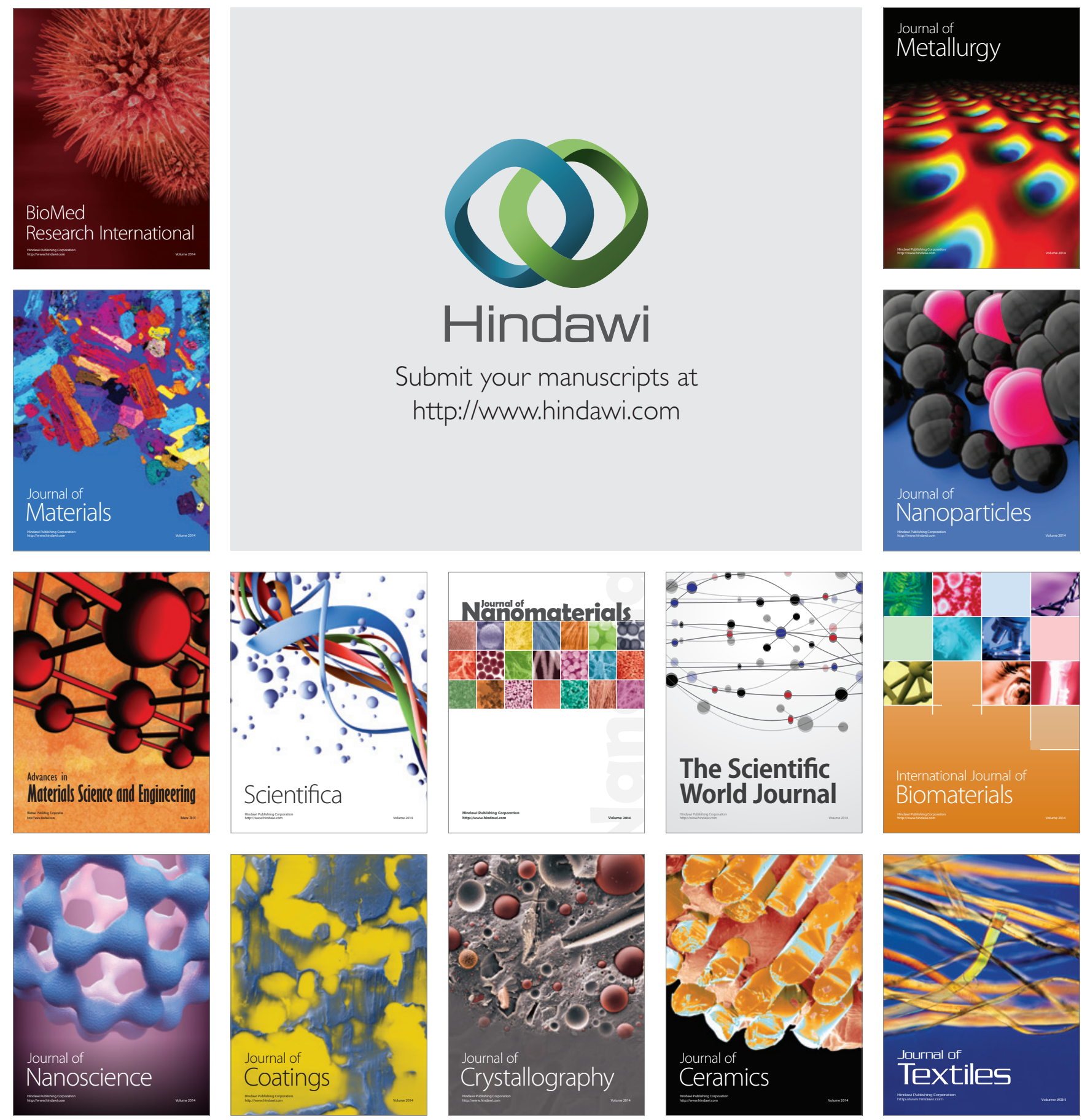